\title{
Individuelle und kantonale Bestimmungsgründe des Zeitaufwands für Hausarbeit von erwerbstätigen Frauen und Männern in der Schweiz
}

\author{
Sarah Kersten* \\ * Departement Sozialwissenschaften, Universität Freiburg, CH-1700 Fribourg, sarah-kersten@gmx.de.
}

\begin{abstract}
s
Individuelle und kantonale Bestimmungsgründe des Zeitaufwands für Hausarbeit von erwerbstätigen Frauen und Männern in der Schweiz
\end{abstract}

Dieser Artikel integriert zum ersten Mal individuelle sowie kantonal differierende politische, kulturelle und wirtschaftliche Bestimmungsgründe zur Analyse des Zeitaufwands für Hausarbeit von erwerbstätigen Frauen und Männern in Paarhaushalten. Die Analyse verweist auf die Bedeutung der Hausarbeit als wichtiger Indikator für Geschlechtergleichheit, neben der Erwerbsarbeit. Obwohl beide Dimensionen reziprok sind, lässt sich zunehmende Gleichstellung auf dem Arbeitsmarkt nicht per se auf die Arbeitsteilung im Haushalt übertragen. Die zugrunde liegenden Zusammenhänge mit kantonalen Kontexten unterscheiden sich.

Schlüsselwörter: Hausarbeit, Schweizer Kantone, geschlechtsspezifische Ungleichheiten, Mehrebenenanalyse, Zeitverwendung

Femmes et hommes employés en Suisse et le temps consacré au travail domestique: I'influence des facteurs individuels et cantonaux

Cet article intègre pour la première fois des facteurs individuels et cantonaux de la politique, de la culture et de l'économie dans l'analyse de l'emploi du temps de travail domestique des femmes et des hommes actifs vivant en couple. L'analyse se réfere au travail domestique comme étant un indicateur important de l'égalité de genre, en plus du travail rémunéré. Bien que les deux dimensions soient liées, une croissance de l'égalité sur le marché du travail ne se répercute pas automatiquement sur la division du travail domestique. Les relations causales different selon les contextes cantonaux.

Mots-clés: Travail domestique, cantons suisses, inégalité de genre, analyse multiniveau, emploi du temps

\section{Individual and Cantonal Factors Explaining Gender Specific Time Use for Housework of Employed Women and Men in Switzerland}

This article combines for the first time individual and cantonal variations of political, cultural and economic factors in order to analyze the individual time use for homework of employed Swiss women and men, living in a partnership. The analysis refers to the significance of housework as indicator for gender equality, in addition to employment. Although both spheres are reciprocal, increasing gender equality on the employment market is not automatically transmitted to the distribution of housework. The underlying correlations with cantonal contexts differ.

Keywords: Homework, Swiss cantons, gender inequality, multilevel analysis, time use 


\title{
Individuelle und kantonale Bestimmungsgründe des Zeitaufwands für Hausarbeit von erwerbstätigen Frauen und Männern in der Schweiz
}

\author{
Sarah Kersten*
}

\section{$1 \quad$ Einleitung}

Die Arbeitsmarktbeteiligung von Frauen ist in den letzten Jahrzehnten stark angestiegen. Dennoch leisten Frauen weitaus mehr Hausarbeit als Männer, selbst bei gleichem Erwerbspensum. Zwar haben Männer in industrialisierten Ländern in diesem Zeitraum durchaus ihren Zeitaufwand für Hausarbeit erhöht und die Geschlechterdifferenzen haben sich verringert, aber es kann kaum von einer Angleichung gesprochen werden (Gershuny und Sullivan 2003, 207). Zum Teil sind die geringer werdenden Geschlechterdifferenzen auch auf den technologischen Fortschritt zurückzuführen, welcher Frauen eine Verringerung ihres Zeitaufwands für Hausarbeit ermöglichte (Cooke 2011). Viele erwerbstätige Frauen sind dennoch einer Doppelbelastung durch Erwerbsarbeit und Hausarbeit ausgesetzt (Treas und Drobnič 2010), die von ihnen oftmals auch als solche empfunden wird (Hans Böckler Stiftung 2014). Der geschlechtsspezifische Zeitaufwand für Hausarbeit kann daher auch als Indikator für das Ausmass der Geschlechtergleichheit einer Gesellschaft betrachtet werden, da die Fortschritte auf dem Arbeitsmarkt nur eine Seite der Medaille sind.

Im Ländervergleich zeigen sich erhebliche Differenzen im Zeitaufwand für Hausarbeit. Die Unterschiede zwischen den Geschlechtern waren Ende der 90er-Jahre in nordeuropäischen Staaten vergleichsweise klein, in Deutschland oder Australien eher hoch und in angelsächsischen Ländern wie Grossbritannien lagen sie im Mittelfeld (Sayer 2010, 34). Aufgrund der grossen Länderunterschiede betonen Studien zur geschlechtlichen Arbeitsteilung von Arbeit im Haushalt und der Familie neben individuellen Faktoren daher die Wichtigkeit von staatlichen und gesellschaftlichen Rahmenbedingungen (Treas und Drobnič 2010; van der Lippe et al. 2011).

Die Schweiz ist eines der föderalsten Länder der Welt. Zwischen den Kantonen gibt es grosse Unterschiede in den sozialpolitischen (Armingeon et al. 2004), wirtschaftlichen (Buchmann 2002) und politisch-institutionellen Profilen (Vatter 2002). Auch hinsichtlich kultureller Aspekte kann die Schweiz nicht als eine Einheit betrachtet werden. So gibt es bei Abstimmungen regelmässig Unterschiede zwischen den Sprachregionen (Bühler 2002), und auch geschlechterkulturelle Leitbilder sind

Departement Sozialwissenschaften, Universität Freiburg, CH-1700 Fribourg, sarah-kersten@ gmx.de. 
unterschiedlich stark verbreitet (Pfau-Effinger 2005). So ist in deutschsprachigen Kantonen das traditionelle männliche Alleinverdienermodell sehr weit verbreitet, während das Doppelverdienermodell stärker in der französisch- und italienischsprachigen Schweiz vertreten ist (Bühler und Meier Kruker 2002; Pfau-Effinger 2005).

Diese kantonalen Unterschiede spiegeln sich auch in der Zeitaufteilung zwischen Erwerbsarbeit und Haus- und Familienarbeit wider. Einer egalitären Zeitaufteilung kommen dabei Genf und Basel-Stadt am nächsten, ländliche Kantone der Deutschschweiz sind hingegen am entferntesten davon (Gasser et al. 2015). Zwei Studien zeigen darüber hinaus, dass Väter in französischsprachigen Kantonen mehr Familienarbeit leisten (Schön-Bühlmann und Liechti 2013; Gasser und Kersten 2015). Epple et al. (2014) identifizieren unterschiedliche Kontextfaktoren als relevant zur Erklärung kantonaler Differenzen der geschlechtsspezifischen Zeitaufteilung. So geht ein traditioneller Kontext mit hoher Zeitungleichheit einher, hohe Wohlfahrtsausgaben, ein schwach ausgeprägter Traditionalismus und ein grosser öffentlicher Sektor hingegen mit geringeren Geschlechterdifferenzen.

Vor diesem Hintergrund stellt sich die Frage, welche Mechanismen in den Schweizer Kantonen eher eine traditionelle Aufteilung der Hausarbeit fördern und in welchen Kontexten sich die Geschlechterunterschiede im Zeitaufwand für Hausarbeit reduzieren. Anhand von Mehrebenenanalysen wird deshalb untersucht, wie neben individuellen auch kantonale Faktoren aus Politik, Kultur und Wirtschaft den individuellen Zeitaufwand für Hausarbeit von in einer Partnerschaft lebenden erwerbstätigen Frauen und Männern beeinflussen.

\section{Eine empirische und theoretische Einordnung}

Das folgende Kapitel legt die theoretischen und empirischen Bezugspunkte auf der individuellen und kantonalen Ebene dar.

\subsection{Individuelle Faktoren}

Theoretische Ansätze zur geschlechtlichen Arbeitsteilung auf Mikro-Ebene können in eine ökonomische und eine Geschlechterperspektive unterschieden werden. Die ökonomische Perspektive zur Erklärung der Aufteilung von Hausarbeit unterstellt den Akteuren rationales Handeln. Als Weiterentwicklung von Beckers (1991) ökonomischer Theorie der Familie, die rein von der Nutzenmaximierung des Haushalts ausgeht, gilt der Ansatz der relativen Ressourcen. Er unterstellt den Akteuren zusätzlich Eigeninteresse und eine Abneigung gegenüber Hausarbeit (Coltrane 2000). Die Verteilung der Hausarbeit ist demnach machtgesteuert; derjenige Partner, der innerhalb des Haushaltes über mehr Ressourcen verfügt, kann den grösseren Teil der Arbeit an den anderen Partner abschieben. Wichtige Ressourcen sind dabei das relative Einkommen, der Bildungsstand und die Berufsposition. Eine weitere ebenfalls 
geschlechtsneutrale Variante ist der Ansatz der Zeitverfügbarkeit, wonach sich die verfügbare Zeit für Hausarbeit nach dem Erwerbspensum richtet (Coverman 1985). Darüber hinaus hängt diese vom Bedarf an Hausarbeit ab, welcher beispielsweise durch die Wohnungsgrösse, einer externen Haushaltshilfe oder durch das Vorhandensein von Kindern beeinflusst wird (Künzler et al. 2001). Eine geschlechtersensible Perspektive nimmt hingegen der "doing gender" Ansatz ein. Er betont die bipolare Orientierung zwischen traditionellen und modernen Einstellungen, die durch Sozialisation erlernt wird und das Verhalten bezüglich der Arbeitsteilung zwischen Paaren beeinflusst (Coltrane 2000). Allerdings werden erst durch die Ausübung von Handlungen selbst Geschlechtsrollen produziert (West und Zimmerman 1987). Demnach ist die geschlechtsspezifische Identität flexibel und relativ zur Situation.

In der Forschung haben sich folgende individuelle Faktoren als besonders einflussreich hinsichtlich des Zeitaufwands für Hausarbeit herausgestellt: Erwerbspensum, Bildung und Familiensituation (Sayer 2010, 20). Dennoch herrscht kein Konsens hinsichtlich der Ergebnisse und ihrer Erklärungskraft, was auch auf Unterschiede in den jeweils zugrunde liegenden Datensätzen zurückzuführen ist (Bianchi und Milkie 2010). ${ }^{1}$

Das eigene Erwerbspensum erweist sich in den meisten Studien als stark hemmender Faktor für den Zeitaufwand für Hausarbeit von Frauen (Künzler et al. 2001) und Männern (Bonoli und Gay-des Cômbes 2005). Hinsichtlich des Einflusses der Erwerbstätigkeit der PartnerInnen gibt es aber widersprüchliche Ergebnisse. Im Einklang mit den Ansätzen der Zeitverfügbarkeit und relativen Ressourcen wird für Frauen zwar einhellig ein Anstieg ihrer Hausarbeitszeit bei steigendem Erwerbspensum ihrer Partner festgestellt (Bianchi et al. 2000; Bonoli und Gay-des Cômbes 2005). Doch nicht alle Studien postulieren für Männer den gleichen Zusammenhang. So stellen einige Studien eine Re-Traditionalisierung der Aufgabenteilung mit steigendem Einkommen der Partnerin fest (Davis et al. 2007). Dies lässt sich über den Ansatz der Geschlechtsrollen erklären, wonach Männer in dieser Situation wahrscheinlich deshalb weniger Hausarbeit verrichten, um ihre Geschlechtsrollenidentität aufrecht zu erhalten. Auch hinsichtlich des Effekts von Bildung gibt es unterschiedliche Ergebnisse. So stellt Gupta (2007) für die USA keinen Effekt der eigenen Bildung auf den Zeitaufwand für Hausarbeit von verheirateten Frauen fest, hingegen verringert sich dieser mit steigendem Bildungsniveau des Partners. Dahingegen stellen Künzler et al. (2001) mit Daten für Deutschland einen negativen Effekt des Bildungsniveaus auf die Hausarbeit von Frauen fest, jedoch keinen Effekt auf die Hausarbeit von Männern.

$1 \quad$ Ein weiteres Problem der Vergleichbarkeit der Studien ist die unterschiedliche Operationalisierung der abhängigen Variable. So wird oftmals nicht zwischen Haus- und Familienarbeit unterschieden, sondern allgemein unbezahlte Arbeit untersucht, wodurch auch die Ergebnisse variieren können. Allerdings unterscheiden sich die Erklärungsmuster für Familienarbeit einerseits, und Hausarbeit andererseits, weshalb eine getrennte Analyse angemessen ist (Sullivan 2013). 


\subsection{Hausarbeit im Kontext: Politik, Kultur und Wirtschaft}

In komparativen Analysen der geschlechtsspezifischen Zeitallokation von Hausarbeit sind die Dimensionen Politik und Kultur die am häufigsten untersuchten Kontextfaktoren (Gershuny und Sullivan 2003; Fuwa 2004; Geist 2005; Stier und Lewin-Epstein 2007; Cooke 2007; Davis et al. 2007; Gupta et al. 2010; van der Lippe et al. 2011). Diese Analysen variieren sowohl hinsichtlich der untersuchten Länder als auch der verwendeten Daten und Indikatoren. Allen gemeinsam ist, dass sich die Kategorie Geschlecht als massgeblich hinsichtlich der Zeitaufteilung für Hausarbeit erweist. Wie gross der moderierende Effekt von Geschlecht ausfällt, hängt allerdings davon ab, inwieweit verschiedene politische und kulturelle Institutionen die Geschlechtergleichheit fördern (Stier und Lewin-Epstein 2007, 239). Dabei wird unisono ein positiver Zusammenhang postuliert: Je höher die Geschlechtergleichheit innerhalb dieser Institutionen, desto eher findet sich auch auf der Mikro-Ebene eine eher egalitäre Aufteilung von Hausarbeit zwischen Frauen und Männern (Batalova und Cohen 2002; Gupta et al. 2010). Als egalitär wird dabei eine Reduktion des Zeitaufwands für Hausarbeit von Frauen und eine Erhöhung des Zeitaufwands von Männern definiert.

Der Einfluss von Politik wird zumeist anhand der Wohlfahrtstypologie von Esping-Andersen (1990) gemessen (Fuwa 2004; van der Lippe et al. 2011). In einer vergleichenden Länderstudie mit Daten von 1994 zeigt beispielsweise Geist (2005), dass in Ländern des konservativen Wohlfahrtstypus die Hausarbeit eher selten gleichwertig zwischen den Partnern aufgeteilt wird, in sozialdemokratischen Ländern hingegen häufiger. Diese Resultate bleiben auch nach Kontrolle auf die drei mikrotheoretischen Ansätze bestehen. Dahingegen stellen Gershuny und Sullivan (2003) bei der Analyse von Daten der Jahre 1960 bis 1990 keinen vergleichbaren Regime-Effekt auf den individuellen Zeitaufwand für Hausarbeit fest.

Die Messung der Dimension Politik anhand der Wohlfahrtsregimetypologie hat den Nachteil, dass die verschiedenen Einflussebenen von Politik ignoriert werden (Stadelmann-Steffen 2007). Die aus den Politikwissenschaften stammende Unterscheidung von Politik in Ergebnisse des politischen Prozesses, den direkten politischen Massnahmen und der prozeduralen Dimension, etwa politische Machtverhältnisse, trägt dieser Kritik Rechnung. ${ }^{2}$

Von konkreten politischen Massnahmen, die auf die Vereinbarkeit von Familie und Beruf abzielen, sind nach Stier und Lewin-Epstein (2007) zwei gegenläufige Mechanismen hinsichtlich des Zusammenhangs mit der geschlechtsspezifischen Verteilung von Hausarbeit zu erwarten. Zum einen führen sie aus, dass Massnahmen zur Erhöhung der Frauenerwerbstätigkeit die Abhängigkeit der Frauen von ihrem Partner reduziert und sich so das Machtverhältnis innerhalb des Haushalts verändert,

2 Siehe auch die aus der angloamerikanischen Politikwissenschaft stammende Unterscheidung des Begriffs Politik in Policies, Politics und Polities (Schmidt 1997) und die schweizerische Studie zu kantonalen Unterschieden der Frauenerwerbstätigkeit von Stadelmann-Steffen (2007). 
was schliesslich zu einer eher egalitären Verteilung der Hausarbeit führen könnte. Diese Annahme geht einher mit dem geschlechtsneutralen Ansatz der relativen Ressourcen. Zum anderen legen sie dar, dass diese politischen Massnahmen auch in keinem Zusammenhang mit der Aufteilung der Hausarbeit stehen könnten (vgl. Gershuny und Sullivan 2003). Da sie oftmals aus flexiblen Arbeitszeiten, Teilzeitbeschäftigung oder grosszügigen Transferzahlungen bestehen, haben Frauen noch immer ausreichend Zeit zur Verfügung, um den Hauptanteil der Hausarbeit zu übernehmen, so dass die Ungleichheiten bestehen bleiben.

In ihrer Analyse können sie keinen Effekt der untersuchten politischen Massnahmen ${ }^{3}$ auf den Zeitaufwand für Hausarbeit von Frauen und Männern feststellen. Sie argumentieren, dass diese nicht auf die Verringerung der Geschlechterungleichheit abzielen, sondern eine Balance zwischen Erwerbsarbeit und Familienleben beabsichtigen. Dies ist in der Mehrheit aber ein Vereinbarkeitsproblem von Frauen, so dass ihnen zwar eine Erwerbstätigkeit ermöglicht wird, ohne dabei aber die Verantwortlichkeit für die Haus- und Familienarbeit, und damit die Beteiligung der Männer, zu beeinflussen.

Wie oben erwähnt, wird die Geschlechtergleichheit auf der politischen Ebene auch durch die prozedurale Dimension der Politik bestimmt, wie etwa Machtverhältnisse verschiedener Akteure. Dieser Faktor wird in Studien zur Aufteilung der Hausarbeit bislang nicht explizit integriert, obschon er in der Literatur zur Ungleichheit der Geschlechter oder in Gender-Regime-Typologien eine wichtige Rolle spielt (Cyba 2000; Walby 2004). Gemäss Korpi (1983) strukturieren politische Kräfteverhältnisse soziale Ungleichheiten, indem die Verteilung der politischen Machtressourcen über die Verteilung von Privilegien entscheidet. In diesem Sinne lässt sich auch die Persistenz geschlechtsspezifischer Lohnungleichheiten, traditionellbürgerlicher Familienmodelle oder die Arbeitsmarktsegregation als Nachhall der historisch tradierten Ausschliessung der Frauen von der Ausübung bürgerlicher, politischer und sozialer Rechte interpretieren (Cyba 2000). Es ist davon auszugehen, dass Ungleichheiten in der geschlechtsspezifischen Verteilung der Arbeit abnehmen beziehungsweise geringer sind, wenn es Frauen besser gelingt, ihre Interessen im politischen Prozess durch stärkere Präsenz durchzusetzen (Phillips 1995). Das Ausmass dieses Ausschlusses lässt sich dementsprechend als Indikator für das Niveau an Geschlechterungleichheit interpretieren (Inglehart und Norris 2003).

Die prozedurale Dimension der Politik wird von Fuwa (2004) in einer Analyse des Zeitaufwands für Hausarbeit mit der Verwendung des Gender-EmpowermentMeasure verwendet. Der Indikator misst Geschlechtergleichheit anhand der politischen und ökonomischen Partizipation und Machtgleichstellung von Frauen (UNDP

3 Stier und Lewin-Epstein $(2007,241)$ konstruieren in Anlehnung an Gornick und Meyers (2004) einen Index aus drei Variablen, um die Vielschichtigkeit von politischen Massnahmen zur Vereinbarkeit von Familie und Beruf zu messen. Diese Variablen sind: Anzahl der Wochen des Mutterschaftsurlaubs bei voller Bezahlung, Anteil an Kleinkindern 0-2 Jahre in öffentlicher Betreuung und die Möglichkeit eines Vaterschaftsurlaubs. 
2009). ${ }^{4}$ Sie zeigt, dass individuelle Verhaltensweisen, die eine egalitäre Aufteilung der Hausarbeit zwischen Paaren anstreben, in Ländern mit einem höheren GenderEmpowerment-Measure erfolgreicher sind. In Ländern mit einem geringeren Mass an Geschlechtergleichheit auf der Länder-Ebene ist die Arbeitsteilung im Haushalt selbst dann weniger egalitär, wenn Frauen in Vollzeit erwerbstätig sind und/oder individuelle Einstellungen zu Geschlechterrollen von Frauen und Männern modern sind. In eher traditionellen Ländern leisten darüber hinaus verheiratete Frauen deutlich mehr Hausarbeit als nicht verheiratete.

Die politische Ausgestaltung eines Landes ist eng mit dominanten kulturellen Werten verknüpft. Die sogenannte Geschlechterkultur (Pfau-Effinger 2000) beschreibt dabei Leitbilder zur geschlechtlichen Verteilung der Erwerbsarbeit und Hausarbeit, die anhand von Normen im institutionellen System verankert ist. So hat die Geschlechterkultur einen direkten Einfluss auf Individuen, indem sich Frauen und Männer in ihrem Handeln an den Leitbildern orientieren. Politische, gesellschaftliche und individuelle Akteure orientieren sich in ihrem Handeln an den Leitbildern, können diese aber ebenfalls transformieren. Pfau-Effinger (2000) unterscheidet verschiedene in Europa dominante Familienmodelle. Das traditionelle Vereinbarkeitsmodell der männlichen Versorgerehe ist beispielsweise in (West)Deutschland stark verbreitet, das Doppelversorgermodell mit einer eher egalitären Aufgabenteilung hingegen in Frankreich oder Finnland.

In einer Analyse zum Zeitaufwand für bezahlte und unbezahlte Arbeit zeigen van der Lippe et al. (2011), dass besonders die landeseigene Kultur den geschlechtsspezifischen Zeitaufwand für Hausarbeit beeinflusst, ökonomische Faktoren hingegen die Erwerbsarbeit. Die Studie betont darüber hinaus auch das Zusammenspiel individueller Charakteristika mit verschiedenen Makro-Faktoren: In Ländern mit einem hohen Maskulinitätsindex ${ }^{5}$ etwa leisten vor allem verheiratete Frauen die meiste Hausarbeit (van der Lippe et al. 2011, 177). Auch Knudsen und Wærness (2008) zeigen, dass die Beteiligung an der Hausarbeit von (Ehe)Frauen von dem Ausmass der Geschlechtergleichheit auf der kulturellen Ebene beeinflusst wird.

An dieser Stelle ist anzumerken, dass der kausale Zusammenhang zwischen den kantonalen Indikatoren für gleichstellungsfördernde oder -ablehnende Kontexte und dem individuellen Zeitaufwand für Hausarbeit und der geschlechtsspezifischen Arbeitsteilung reziprok sein kann. So sind in dieser Studie jeweils die Ursachen nicht zu benennen, sondern nur Zusammenhänge identifizierbar.

Schliesslich stellt die Wirtschaft eines Landes die Rahmenbedingungen dar, innerhalb welcher Politik sowie individuelles Handeln verläuft. Je nach Wirtschaftsstruktur und Modernisierungsgrad gestalten sich diese unterschiedlich. Diese Faktoren stehen aber eher in einem indirekten Zusammenhang mit der geschlechtsspezifischen

4 Allerdings verortet Fuwa (2004) diesen Indikator nicht in der Politik, sondern der Kultur eines Landes.

5 Der Maskulinitätsindex wurde auf Basis von Hofstede (2001) erstellt und misst, wie präsent die klassische geschlechtsspezifische Rollenverteilung in einer Gesellschaft ist. 
Verteilung von Hausarbeit (Fuwa 2004; Knudsen und Wærness 2008). Eine prosperierende Wirtschaft beispielsweise stimuliert die Erwerbstätigkeit von Frauen und Männern (van der Lippe et al. 2011). Die Rahmenbedingungen schaffen Anreize für Frauen, wirtschaftlich unabhängig zu sein (zum Zusammenhang von Frauenerwerbstätigkeit und wirtschaftlichen Faktoren in der Schweiz vgl. Stadelmann-Steffen 2007 und Buchmann et al. 2010). In Anlehnung an die oben erläuterten Mikro-Theorien führt dies aber nicht per se zu einer egalitären Aufteilung der Hausarbeit. Vor dem Hintergrund der anhaltenden Bedeutung von traditionellen Geschlechterrollen in industrialisierten Gesellschaften argumentieren Knudsen und Wærness (2008) dafür, dass die Hausarbeit von Frauen sehr viel eher von weiblichem Empowerment auf der politischen Ebene, während die von Männern stärker durch die wirtschaftliche Ebene beeinflusst wird.

Insgesamt zeichnet sich ein komplexes Bild des Zusammenhangs von Politik, Kultur und Wirtschaft sowie individuellen Faktoren mit dem geschlechtsspezifischen Zeitaufwand für Hausarbeit. Dieses soll im folgenden Abschnitt in einem analytischen Modell verdeutlicht werden.

Der individuelle Zeitaufwand für Hausarbeit ist die zu untersuchende Variable. Es werden sowohl Effekte auf der individuellen als auch auf der kantonalen, beziehungsweise Kontextebene analysiert.

Auf der Individualebene werden Hypothesen anhand des Ansatzes der Zeitverfügbarkeit, der relativen Ressourcen und Geschlechtsrollen aufgestellt. Nach dem Ansatz der Zeitverfügbarkeit wird erwartet, dass der Zeitaufwand für Hausarbeit mit steigendem Erwerbspensum sinkt, unabhängig des Geschlechts. Ein Kind erhöht den Bedarf an Hausarbeit massgeblich. Daher wird erwartet, dass ab einem Kind im Haushalt bei Frauen und Männern der Zeitaufwand für Hausarbeit steigt. Eine externe Haushaltshilfe hingegen reduziert den eigenen Aufwand für Hausarbeit, weshalb angenommen wird, dass sich bei Vorhandensein einer externen Haushaltshilfe bei beiden Geschlechtern der Zeitaufwand für Hausarbeit reduziert. Das Bildungsniveau sollte nach dem Ansatz der relativen Ressourcen einen senkenden Effekt auf die Hausarbeit haben, unabhängig vom Geschlecht. Hingegen sollte sich mit steigendem Bildungsniveau und steigendem Erwerbspensum der PartnerInnen der eigene Aufwand für Hausarbeit erhöhen. ${ }^{6}$ Allerdings können dem

6 Um den Ansatz der relativen Ressourcen zu überprüfen, wird in der Regel das Einkommen herangezogen. Mit dem für diese Analyse vorliegenden Datensatz (Schweizerische Arbeitskräfteerhebung SAKE) ist dies aber aufgrund zu vieler fehlender Fälle nicht möglich, weshalb hier auch auf die theoretischen Zusammenhänge nicht eingegangen wird. Weiter wird der Ansatz der relativen Ressourcen nicht mit dem relativen Bildungsniveau und Erwerbspensum der (Ehe) Partner überprüft. Die dritte Einschränkung bei der Analyse der mikrotheoretischen Ansätze 
Geschlechtsrollenansatz zufolge hier auch geschlechtsspezifische Effekte erwartet werden. Demnach müsste der Zeitaufwand für Hausarbeit von Männern sinken, arbeitet ihre Partnerin in Vollzeit.

Auf der Kontextebene werden die Effekte der Dimensionen Politik, Kultur und Wirtschaft analysiert. Von den Indikatoren der Politik und Kultur wird erwartet, dass sie einen geschlechteregalisierenden Effekt auf den Zeitaufwand für Hausarbeit haben, umso höher ihr Niveau an Geschlechtergleichheit auf der kantonalen Ebene ist. Konkret wird mit steigendem kantonalen Versorgungsgrad an Kinderbetreuungsplätzen erwartet, dass sich der Zeitaufwand für Hausarbeit von Männern erhöht und jener von Frauen sinkt. Wie oben erläutert, kann die Hypothese für den $\mathrm{Zu}$ sammenhang politischer Massnahmen und der individuellen Zeitverwendung von Frauen auch kontradiktorisch lauten. So ist es umstritten, inwieweit sich der positive Zusammenhang zwischen gleichstellungspolitischen Massnahmen zur Erhöhung der Frauenerwerbstätigkeit in einen egalisierenden Effekt hinsichtlich der Aufteilung der Hausarbeit übertragen lässt. Entsprechend kann also die Alternativhypothese aufgestellt werden, wonach erwartet wird, dass sich der Zeitaufwand für Hausarbeit von Frauen mit steigendem Versorgungsgrad an Kinderbetreuungsplätzen nicht reduziert. Weiter wird angenommen, dass in Kantonen mit hohem Frauenanteil im Parlament Männer eher mehr und Frauen eher weniger Hausarbeit leisten, da ein hoher Frauenanteil im Parlament als ein gleichstellungsfördernder Kontext betrachtet wird.

Im Gegensatz zu den oben zitierten internationalen Studien gibt es für die Schweiz keine Daten zu Gleichstellungsindizes auf der kantonalen Ebene, wie beispielsweise dem Gender-Empowerment-Measure. Für die Kultur werden daher zwei Faktoren verwendet, die als Proxy-Variablen für die Geschlechtergleichheit auf der kantonalen Ebene zu verstehen sind: die Sprachregion, gemessen am Anteil der deutschsprachigen Bevölkerung, und ein Index für Traditionalismus. Ein starker Traditionalismus kann als eine Ablehnung von jeglicher kultureller Veränderung verstanden werden, einschliesslich von Veränderungen der Geschlechterverhältnisse und zunehmender Geschlechtergleichheit (Stolz 2001). Cyba (2000, 140 ff.) versteht Traditionalismus als einen spezifischen Aspekt sozialer Institutionen und Organisationen, der als Gegensatz zur Moderne Veränderungen ablehnt und sich auf das immer schon Gewesene beruft.

In Anlehnung an Bühler und Meier Kruker (2002) und Pfau-Effinger (2005) wird erwartet, dass in deutschsprachigen Kantonen die Aufteilung der Arbeit geschlechtsspezifisch erfolgt und somit Frauen eher mehr und Männer eher weniger Hausarbeit leisten. Dieser Zusammenhang wird auch für traditionelle Kantone erwartet.

betrifft den "doing gender" Ansatz, der nur indirekt überprüft werden kann, weil in der SAKE keine Einstellungsvariablen zu den Geschlechterrollen erhoben werden. 
Von den Wirtschaftsfaktoren schliesslich wird die Hypothese formuliert, dass ein prosperierender Wirtschaftskontext in Form einer hohen Beschäftigtenrate und einem hohen Urbanisierungsgrad zu einer Reduzierung des Zeitaufwands für Hausarbeit von Frauen und einer Erhöhung desjenigen von Männern führt.

Die drei hier betrachteten Kontextdimensionen sind interdependent. Somit vereinfacht die Herangehensweise dieser Analyse die Realität erheblich, indem die kausalen Zusammenhänge zwischen Politik, Kultur und Wirtschaft nicht analysiert werden. Die empirischen Restriktionen, auf die im Folgenden eingegangen wird, erlauben keine andere Herangehensweise.

\section{Daten und Methode}

\subsection{Daten}

Die Analyse wird mit Daten der Schweizerischen Arbeitskräfteerhebung (SAKE) aus dem Jahr 2010 durchgeführt. Die SAKE befragt jährlich Personen ab 15 Jahren mit schweizerischer Nationalität sowie ausländischer Staatsangehörigkeit, die seit mindestens einem Jahr in der Schweiz leben. Der Auswahlplan für die Telefoninterviews beruht auf einer nach Kantonen geschichteten Zufallsstichprobe und erfolgt daher proportional zur Bevölkerungsgrösse der Kantone. Es werden nur erwerbstätige Frauen und Männer zwischen 15 und 64 Jahren untersucht, die mit einem Partner beziehungsweise einer Partnerin im gleichen Haushalt leben. Als erwerbstätig gelten dabei Personen, die in der Referenzwoche mindestens eine Stunde gegen Bezahlung in einem selbstständigen oder unselbstständigen Beschäftigungsverhältnis standen oder unentgeltlich im Familienbetrieb gearbeitet haben. Dazu zählt auch die vorübergehende Abwesenheit vom Arbeitsplatz aufgrund von Krankheit, Urlaub oder Ähnlichem.

Der Aufwand für Hausarbeit an Wochenenden ist - gerade bei Berufstätigen ungleich viel höher als unter der Woche (Bühlmann und Schmid 1999). Deshalb werden nur diejenigen Befragten in die Stichprobe mit aufgenommen, die zu einem Wochentag nach ihrem Zeitaufwand für Hausarbeit befragt wurden (entspricht 37\% der Stichprobe). Somit befinden sich insgesamt 2194 Frauen und 2666 Männer in der Stichprobe.

Die abhängige Variable ist der Zeitaufwand für Hausarbeit, in Stunden pro Werktag. Im Modul «Unbezahlte Arbeit» der SAKE werden acht Kategorien zum Zeitaufwand für Hausarbeit erfasst. Diese sind: Mahlzeiten zubereiten; Abwaschen, Einräumen, Tisch decken; Einkaufen; Putzen, Aufräumen; Waschen, Bügeln; Handwerkliche Tätigkeiten, Handarbeiten; Haustiere, Pflanzen, Garten und administrative Arbeiten. Zur Erfassung wird die Referenztagmethode verwendet, d. h. die Fragen beziehen sich auf einen bestimmten Referenztag in der Woche, meist den Vortag. 


\section{Tabelle 1 Operationalisierung der unabhängigen Variablen}

\begin{tabular}{|c|c|}
\hline Variablen & Operationalisierung \\
\hline \multicolumn{2}{|l|}{ Individualebene } \\
\hline \multicolumn{2}{|l|}{ Mikro-theoretische Ansätze } \\
\hline Erwerbsarbeitsstunden & Wöchentliche Arbeitszeit, in Stunden pro Woche; metrisch erfasst. \\
\hline Tertiärbildung & Höchste abgeschlossene Ausbildung: Tertiärstufe $=1$; Sekundarstufe I oder $\|=0$. \\
\hline Teilzeit Partnerln & $\begin{array}{l}\text { Dummy: Beschäftigungsgrad des/der PartnerIn kleiner als } 90 \%=1 ; \text { Partnerln } \\
\text { nicht erwerbstätig, Beschäftigungsgrad des/der PartnerIn grösser als } 90 \%=0 \text {. }\end{array}$ \\
\hline Vollzeit Partnerln & $\begin{array}{l}\text { Dummy: Beschäftigungsgrad des/der Partnerln grösser als } 90 \%=1 ; \text { Partnerln } \\
\text { nicht erwerbstätig, Beschäftigungsgrad des/der Partnerln kleiner als } 90 \%=0 \text {. }\end{array}$ \\
\hline Tertiärbildung Partnerln & $\begin{array}{l}\text { Dummy: höchste abgeschlossene Ausbildung des/der Partnerln: Tertiärstufe = 1; } \\
\text { Sekundarstufe I oder } \|=0 \text {. }\end{array}$ \\
\hline Kind & $\begin{array}{l}\text { Dummy: mindestens ein Kind zwischen 0-6 Jahren im Haushalt }=1 \text {; } \\
\text { kein Kind unter } 6 \text { Jahren }=0 \text {. }\end{array}$ \\
\hline Externe Hilfe & $\begin{array}{l}\text { Dummy: Nutzung einer externen Haushaltshilfe }=1 \text {; keine externe Haushalts- } \\
\text { hilfe }=0 \text {. }\end{array}$ \\
\hline \multicolumn{2}{|l|}{ Kontrollvariablen } \\
\hline Verheiratet & Dummy: Verheiratet $=1$; Ledig, getrennt, verwitwet $=0$. \\
\hline Alter; Alter quadriert & Alter in Jahren, metrisch erfasst. \\
\hline Ausländerln & Dummy: Befragte/r ausländischer Herkunft $=1 ;$ Schweizer $\mid n=0$. \\
\hline \multicolumn{2}{|l|}{ Kantonale Ebene } \\
\hline \multicolumn{2}{|l|}{ Politik } \\
\hline Versorgungsgrad & $\begin{array}{l}\text { Versorgungsgrad mit Ganztagesbetreuungsangeboten (gewichtete Anzahl Be- } \\
\text { treuungsplätze pro im Kanton wohnhaftes Kind) im Frühbereich (Kinder im Alter } \\
\text { von 0-3 Jahren), Jahre 2009/2010; Quelle: Stern et al. (2013). }\end{array}$ \\
\hline $\begin{array}{l}\text { Frauenanteil im } \\
\text { Parlament }\end{array}$ & $\begin{array}{l}\text { Anteil Frauen in den kantonalen Parlamenten, 2009; Quelle: IDHEAP/BADAC } \\
\text { (2014). }\end{array}$ \\
\hline \multicolumn{2}{|l|}{ Kultur } \\
\hline Anteil Deutsch & $\begin{array}{l}\text { Anteil an deutschsprechenden Personen pro Kanton; Quelle: BFS (2010), eigene } \\
\text { Berechnungen. }\end{array}$ \\
\hline Traditionalismus & $\begin{array}{l}\text { Der Index misst die durchschnittlichen Einstellungen der Bevölkerung mit } \\
\text { Stimmberechtigung auf kantonaler Ebene hinsichtlich vier Themen: Erhalt von } \\
\text { Traditionen, starke Armee, Recht und Ordnung und Chancengleichheit für Aus- } \\
\text { länder. Je höher der Wert, desto stärker der Traditionalismus; Quelle: Daten der } \\
\text { Schweizer Wahlstudie Selects aus dem Jahr 2007, basierend auf Berechnungen } \\
\text { von Epple (2013). }\end{array}$ \\
\hline \multicolumn{2}{|l|}{ Wirtschaft } \\
\hline Beschäftigtenquote & $\begin{array}{l}\text { Anteil der Erwerbstätigen an der Bevölkerung im erwerbsfähigen Alter (15-64 } \\
\text { Jahre), 2009; Quelle: BFS (2009), eigene Berechnungen. }\end{array}$ \\
\hline Urbanisierungsgrad & $\begin{array}{l}\text { Anteil der in Agglomerationen oder isolierten Städten wohnhaften Bevölkerung } \\
\text { (Gemeinden mit mehr als } 10000 \text { Einwohnern), 2001; Quelle: IDHEAP/BADAC } \\
\text { (2014). }\end{array}$ \\
\hline
\end{tabular}


Auf der individuellen Ebene werden die drei erläuterten theoretischen Ansätze (Zeitverfügbarkeit, relative Resssourcen, Geschlechtsrollenansatz) anhand folgender unabhängiger Variablen getestet: Erwerbsarbeitsstunden, Bildungsstand, Erwerbspensum $^{7}$, Bildungsstand der PartnerInnen, eine Dummy-Variable für mindestens ein Kind zwischen 0-6 Jahren im Haushalt und eine Dummy-Variable für die Nutzung einer externen Haushaltshilfe. Als Kontrollvariablen werden weiter der Ehestand, das Alter und die Herkunft integriert.

Auf der kantonalen Ebene werden insgesamt sechs Variablen integriert. Innerhalb der Politik wird zum einen der Versorgungsgrad an Kinderbetreuungsplätzen im Frühbereich (für Kinder zwischen 0-3 Jahren) als Indikator für politische Massnahmen integriert. Zum anderen wird der Frauenanteil im Parlament, als Indikator für die prozedurale Dimension der Politik, untersucht. Für die Geschlechterkultur werden die Sprachregion, gemessen am Anteil Deutschsprachiger, und ein Index für Traditionalismus integriert. Schliesslich werden zwei Wirtschaftsfaktoren integriert. Die Beschäftigtenquote dient als Indikator für die konjunkturelle Situation, der $U_{r}$ banisierungsgrad als Indikator für den Grad der Modernisierung (vgl. StadelmannSteffen 2007).

Ausführliche Angaben zur Operationalisierung der unabhängigen Variablen finden sich in Tabelle 1.

\subsection{Methodisches Vorgehen}

Für die Beantwortung der Fragestellung werden Mehrebenenanalysen durchgeführt (Rabe-Hesketh und Skrondal 2005; Hox 2010; Snijders und Bosker 2011). Diese Methode bietet mehrere Vorteile. Zum einen bildet sie die hierarchische Struktur der Daten ab (Individuen sind in Kantone gruppiert), so dass gleichermassen individuelle wie kantonale Faktoren als erklärende Variablen integriert werden können. Durch die Modellierung der Varianz auf der Mikro- und Makroebene kann so gemessen werden, inwieweit sich die Ungleichheiten auf Unterschiede zwischen den Individuen oder auf solche zwischen den Kontextfaktoren beziehen. Dabei lässt es die geringe Anzahl an Kantonen $(\mathrm{N}=26)$ nicht zu, mehr als zwei Kontextfaktoren pro Modell zu schätzen. Deshalb können die drei Kontextdimensionen Politik, Kultur und Wirtschaft nur getrennt getestet werden, und die Annahme ihrer Interdependenz bleibt auf der theoretischen Ebene verhaftet. Zum anderen entspricht das methodische Vorgehen der Mehrebenenanalyse der Annahme, wonach individuelles Handeln kontextabhängig ist. Im Gegensatz zu herkömmlichen Regressionen wird also davon ausgegangen, dass das Niveau der abhängigen Variable über die Kantone variiert. Um die geschlechtsspezifischen Effekte darzustellen, werden für Frauen und Männer getrennte Modelle berechnet.

$7 \quad$ Im Gegensatz zu den Angaben der Befragten erfasst die SAKE das Erwerbspensum der PartnerInnen nur in Stellenprozenten und nicht in Stunden pro Woche. 


\section{Ergebnisse}

Tabelle 2 bildet den kantonalen Zeitaufwand für Hausarbeit von erwerbstätigen Frauen und Männern an Werktagen ab. Im Durchschnitt leisten Männer eine Stunde 47 Minuten Hausarbeit pro Werktag, und Frauen drei Stunden. In allen Kantonen leisten Frauen mehr Hausarbeit als Männer. Besonders gross sind die Geschlechterdifferenzen in den Kantonen Schwyz, Wallis und Thurgau. Hier leisten Frauen täglich über zweieinhalb Stunden mehr Hausarbeit als Männer. Im Tessin sind es sogar fast drei Stunden, die Frauen mehr Hausarbeit leisten als Männer. In den Kantonen Basel-Landschaft, Basel-Stadt, und Waadt sind die Differenzen zwischen dem Zeitaufwand für Hausarbeit von Frauen und Männern vergleichsweise gering: Frauen leisten bis eineinhalb Stunden mehr Hausarbeit als Männer.

Tabelle 2 Durchschnittlicher Zeitaufwand für Hausarbeit, in Stunden pro Werktag, von erwerbstätigen Frauen und Männern und deren Differenz, kantonale Mittelwerte, 2010

\begin{tabular}{lrrr}
\hline & Frauen & Männer & Geschlechterdifferenz \\
\hline Zürich & 1.43 & 3.1 & 1.71 \\
Bern & 1.61 & 3.6 & 2.03 \\
Luzern & 1.60 & 3.9 & 2.28 \\
Schwyz & 1.56 & 3.9 & 2.37 \\
Freiburg & 1.71 & 3.9 & 2.15 \\
Solothurn & 1.69 & 3.6 & 1.95 \\
Basel-Stadt & 2.05 & 3.2 & 1.15 \\
Basel-Landschaft & 2.04 & 3.1 & 1.06 \\
St. Gallen & 1.45 & 3.8 & 2.37 \\
Aargau & 1.80 & 3.9 & 2.06 \\
Thurgau & 1.33 & 3.7 & 2.41 \\
Tessin & 1.51 & 4.4 & 2.91 \\
Waadt & 1.66 & 2.9 & 1.29 \\
Wallis & 1.92 & 4.3 & 2.40 \\
Genf & 1.82 & 3.4 & 1.63 \\
Schweiz & 1.68 & 3.7 & 1.98 \\
\hline
\end{tabular}

Anmerkung: Es werden nur die Kantone dargestellt, deren ungewichtete Anzahl N $>50$ beträgt. In der empirischen Analyse werden Befragte aus Kantonen mit kleinerem N hingegen integriert.

Quelle: Eigene Berechnungen mit Daten der Schweizerischen Arbeitskräfteerhebung 2010 (BFS 2010). 
Tabelle 3 Mehrebenenmodelle zur Erklärung des geschlechtsspezifischen Zeitaufwands für Hausarbeit von Frauen

\begin{tabular}{|c|c|c|c|c|c|}
\hline & (1) & (2) & (3) & (4) & (5) \\
\hline \multicolumn{6}{|l|}{ Individualebene } \\
\hline \multirow[t]{2}{*}{ Alter } & & 0.021 ** & $0.021^{* *}$ & $0.020^{* *}$ & $0.020^{* *}$ \\
\hline & & $(0.007)$ & $(0.007)$ & $(0.007)$ & $(0.007)$ \\
\hline \multirow[t]{2}{*}{ Alter quadriert } & & $-0.001^{*}$ & $-0.001^{*}$ & $-0.001^{*}$ & $-0.001^{*}$ \\
\hline & & $(0.001)$ & $(0.001)$ & $(0.001)$ & $(0.001)$ \\
\hline \multirow[t]{2}{*}{ Verheiratet } & & 0.620 ** & $0.614^{* *}$ & $0.612^{* *}$ & $0.615^{* *}$ \\
\hline & & $(0.151)$ & $(0.151)$ & $(0.151)$ & $(0.151)$ \\
\hline \multirow[t]{2}{*}{ Erwerbsarbeitszeit } & & $-0.050^{* *}$ & $-0.050^{* *}$ & $-0.050^{* *}$ & -0.050 ** \\
\hline & & $(0.004)$ & $(0.004)$ & $(0.004)$ & $(0.004)$ \\
\hline \multirow[t]{2}{*}{ Kind } & & 0.241 & 0.239 & 0.227 & 0.230 \\
\hline & & $(0.154)$ & $(0.154)$ & $(0.154)$ & $(0.154)$ \\
\hline \multirow[t]{2}{*}{ Tertiärstufe } & & $-0.428^{* *}$ & $-0.422^{* *}$ & $-0.423^{* *}$ & $-0.434^{* *}$ \\
\hline & & $(0.139)$ & $(0.139)$ & $(0.139)$ & $(0.139)$ \\
\hline \multirow[t]{2}{*}{ Ausländer } & & 0.066 & 0.071 & 0.077 & 0.080 \\
\hline & & $(0.123)$ & $(0.123)$ & $(0.123)$ & $(0.123)$ \\
\hline \multirow[t]{2}{*}{ Teilzeit Partnerln } & & -0.224 & -0.220 & -0.227 & -0.228 \\
\hline & & $(0.276)$ & $(0.276)$ & $(0.276)$ & $(0.276)$ \\
\hline \multirow[t]{2}{*}{ Vollzeit Partnerln } & & $0.373^{*}$ & $0.373^{*}$ & $0.363^{+}$ & $0.383^{*}$ \\
\hline & & $(0.187)$ & $(0.186)$ & $(0.187)$ & $(0.187)$ \\
\hline \multirow[t]{2}{*}{ Tertiärbildung Partnerln } & & $-0.320^{\star}$ & $-0.311^{*}$ & $-0.304^{*}$ & $-0.298^{*}$ \\
\hline & & $(0.127)$ & $(0.128)$ & $(0.127)$ & $(0.128)$ \\
\hline \multirow[t]{2}{*}{ Externe Hilfe } & & -0.031 & -0.025 & -0.043 & -0.039 \\
\hline & & (0.195) & $(0.195)$ & (0.195) & $(0.195)$ \\
\hline
\end{tabular}

Kontextebene

Versorgungsgrad

$-0.166$

$(1.081)$

Frauenanteil im Parlament $-0.032^{* *}$ (0.012)

Anteil Deutschsprechende $-0.344^{*}$ (0.155) $0.025^{*}$ (0.010)

Beschäftigtenquote

Urbanisierungsgrad $-0.701^{*}$ (0.296) 
Fortsetzung der Tabelle 3.

\begin{tabular}{lccccc}
\hline & $(1)$ & $(2)$ & $(3)$ & $(4)$ & $(5)$ \\
\hline Konstante & $3.616^{* *}$ & $4.434^{* *}$ & $5.272^{* *}$ & $2.842^{* *}$ & $6.964^{* *}$ \\
& $(-0.083)$ & $(0.281)$ & $(0.409)$ & $(0.655)$ & $(1.215)$ \\
Varianz Kontextebene & $0.066^{* *}$ & $0.066^{*}$ & $0.003^{*}$ & $0.003^{*}$ & $0.001^{*}$ \\
Varianz Individualebene & 7.439 & $7.439^{* *}$ & $6.649^{* *}$ & $6.651^{* *}$ & $6.661^{* *}$ \\
$N$ & 2194 & 2194 & 2194 & 2194 & 2194 \\
\hline
\end{tabular}

Anmerkung: ${ }^{*} p \leq 0.01,{ }^{*} p \leq 0.05,{ }^{*} p \leq 0.1$. Standardfehler in Klammern. Nicht-standardisierte b-Koeffizienten. Quelle: Eigene Berechnungen mit Daten der Schweizerischen Arbeitskräfteerhebung 2010 (BFS 2010).

Tabelle 3 und 4 stellen die Mehrebenenmodelle für Frauen und Männer dar. Das Nullmodell (Modell 1 respektive 6) gibt Auskunft darüber, inwiefern die Gesamtvarianz des Zeitaufwands für Hausarbeit auf Unterschiede zwischen den Kantonen zurückzuführen ist (Hox 2010; Snijders und Bosker 2011). Die Schätzungen zeigen, dass eine signifikante Kontextvarianz existiert, daher kann die statistisch zuverlässige Aussage getroffen werden, dass die kantonalen Unterschiede im Zeitaufwand für Hausarbeit nicht zufällig zustande kommen, sondern Folge systematischer kantonaler Differenzen sind, die sich im individuellen Verhalten manifestieren.

Die Modelle 2 und 7 beinhalten nur Individualvariablen. Einige der Kontrollvariablen zeigen lediglich bei Frauen einen signifikanten Zusammenhang. So steigt mit zunehmendem Alter ihr Zeitaufwand für Hausarbeit, nimmt aber schliesslich wieder ab, wie der signifikante Effekt von Alter quadriert zeigt. Darüber hinaus leisten verheiratete Frauen signifikant mehr Hausarbeit als nicht verheiratete Frauen, obschon beide Vergleichsgruppen in einer Partnerschaft leben. Eine Ehe befördert stark die geschlechtsspezifische Aufteilung der Hausarbeit und auch die Ungleichheiten im Zeitaufwand innerhalb der Gruppe der Frauen. Und schliesslich zeigen sich in Bezug auf den Zeitaufwand für Hausarbeit keine signifikanten Unterschiede zwischen AusländerInnen und SchweizerInnen.

Hinsichtlich der Indikatoren für den Ansatz der Zeitverfügbarkeit bestätigt sich die Annahme, wonach eine steigende Erwerbsarbeitszeit mit einer Reduzierung der Hausarbeitszeit einhergeht, unabhängig vom Geschlecht. Ein Kind im Haushalt hingegen erhöht lediglich den Zeitaufwand für Hausarbeit von Männern. So kann die Hypothese für Frauen nicht bestätigt werden. Auch der Zusammenhang zwischen einer externen Haushaltshilfe und der Hausarbeit erfolgt nicht wie erwartet. So zeigt sich kein signifikanter Effekt für Frauen, bei Männern hingegen erhöht sich der Zeitaufwand für Hausarbeit sogar. Insgesamt nehmen nur 10\% der Stichprobe eine externe Haushaltshilfe in Anspruch. 
Tabelle $4 \quad$ Mehrebenenmodelle zur Erklärung des geschlechtsspezifischen Zeitaufwands für Hausarbeit von Männern

\begin{tabular}{|c|c|c|c|c|c|}
\hline & (6) & (7) & (8) & (9) & (10) \\
\hline \multicolumn{6}{|l|}{ Individualebene } \\
\hline Alter & & $\begin{array}{c}-0.004 \\
(0.005)\end{array}$ & $\begin{array}{c}-0.004 \\
(0.005)\end{array}$ & $\begin{array}{c}-0.004 \\
(0.005)\end{array}$ & $\begin{array}{c}-0.004 \\
(0.005)\end{array}$ \\
\hline Alter quadriert & & $\begin{array}{c}0.000 \\
(0.000)\end{array}$ & $\begin{array}{c}0.000 \\
(0.000)\end{array}$ & $\begin{array}{c}0.000 \\
(0.000)\end{array}$ & $\begin{array}{c}0.000 \\
(0.000)\end{array}$ \\
\hline Verheiratet & & $\begin{array}{r}-0.160 \\
(0.100)\end{array}$ & $\begin{array}{r}-0.160 \\
(0.100)\end{array}$ & $\begin{array}{c}-0.164 \\
(0.100)\end{array}$ & $\begin{array}{r}-0.168^{+} \\
(0.100)\end{array}$ \\
\hline Erwerbsarbeitszeit & & $\begin{array}{c}-0.013^{* *} \\
(0.004)\end{array}$ & $\begin{array}{c}-0.013^{* *} \\
(0.004)\end{array}$ & $\begin{array}{c}-0.013^{* *} \\
(0.004)\end{array}$ & $\begin{array}{c}-0.013^{* *} \\
(0.004)\end{array}$ \\
\hline Kind & & $\begin{array}{l}0.226^{* *} \\
(0.087)\end{array}$ & $\begin{array}{l}0.228^{* *} \\
(0.087)\end{array}$ & $\begin{array}{l}0.229 * * \\
(0.087)\end{array}$ & $\begin{array}{l}0.232^{* *} \\
(0.087)\end{array}$ \\
\hline Tertiärstufe & & $\begin{array}{c}-0.071 \\
(0.075)\end{array}$ & $\begin{array}{c}-0.067 \\
(0.075)\end{array}$ & $\begin{array}{c}-0.063 \\
(0.075)\end{array}$ & $\begin{array}{c}-0.059 \\
(0.075)\end{array}$ \\
\hline Ausländer & & $\begin{array}{r}-0.038 \\
(0.070)\end{array}$ & $\begin{array}{c}-0.044 \\
(0.071)\end{array}$ & $\begin{array}{r}-0.046 \\
(0.070)\end{array}$ & $\begin{array}{r}-0.035 \\
(0.071)\end{array}$ \\
\hline Teilzeit Partnerln & & $\begin{array}{c}0.194^{*} \\
(0.079)\end{array}$ & $\begin{array}{c}0.199^{*} \\
(0.079)\end{array}$ & $\begin{array}{c}0.202^{*} \\
(0.079)\end{array}$ & $\begin{array}{c}0.200 * \\
(0.079)\end{array}$ \\
\hline Vollzeit Partnerln & & $\begin{array}{l}0.356 \text { ** } \\
(0.097)\end{array}$ & $\begin{array}{l}0.361 * * \\
(0.097)\end{array}$ & $\begin{array}{l}0.365^{* *} \\
(0.097)\end{array}$ & $\begin{array}{l}0.359 * * \\
(0.097)\end{array}$ \\
\hline Tertiärbildung Partnerln & & $\begin{array}{c}-0.043 \\
(0.087)\end{array}$ & $\begin{array}{c}-0.051 \\
(0.088)\end{array}$ & $\begin{array}{c}-0.060 \\
(0.088)\end{array}$ & $\begin{array}{c}-0.052 \\
(0.088)\end{array}$ \\
\hline Externe Hilfe & & $\begin{array}{c}0.263^{*} \\
(0.118)\end{array}$ & $\begin{array}{c}0.258^{*} \\
(0.118)\end{array}$ & $\begin{array}{c}0.254^{*} \\
(0.118)\end{array}$ & $\begin{array}{c}0.262^{*} \\
(0.118)\end{array}$ \\
\hline
\end{tabular}

Kontextebene

Versorgungsgrad

0.625

(0.665)

$-0.006$

(0.008)

Anteil Deutschsprechende

$-0.198^{*}$

(0.091)

0.002

(0.006)

Beschäftigtenquote

(0.175) 
Fortsetzung der Tabelle 4.

\begin{tabular}{lccccc}
\hline & $(6)$ & $(7)$ & $(8)$ & $(9)$ & $(10)$ \\
\hline Konstante & $1.632^{* *}$ & $2.086^{* *}$ & $2.183^{* *}$ & $1.933^{* *}$ & $4.457^{* *}$ \\
& $(0.034)$ & $(0.202)$ & $(0.279)$ & $(0.410)$ & $(0.735)$ \\
Varianz Kontextebene & $0.003^{*}$ & $0.004^{*}$ & $0.004^{*}$ & $0.001^{*}$ & $0.001^{*}$ \\
Varianz Individualebene & $3.180^{* *}$ & $2.872^{* *}$ & $2.872^{* *}$ & $2.872^{* *}$ & $2.866^{* *}$ \\
$N$ & 2666 & 2666 & 2666 & 2666 & 2666 \\
\hline
\end{tabular}

Anmerkung: ${ }^{* *} p \leq 0.01,{ }^{*} p \leq 0.05,{ }^{+} p \leq 0.1$. Standardfehler in Klammern. Nicht-standardisierte b-Koeffizienten. Quelle: Eigene Berechnungen mit Daten der Schweizerischen Arbeitskräfteerhebung 2010 (BFS 2010).

Frauen mit Tertiärbildung wenden eher weniger Zeit für Hausarbeit auf als Frauen mit niedrigerem Bildungsniveau, womit sich für sie die Hypothese der relativen Ressourcen bestätigt. Für Männer zeigt sich hingegen kein Zusammenhang. Insgesamt wiederholt sich hier möglicherweise der Zeitverfügbarkeitseffekt, da höher gebildete Frauen in der Regel auch ein höheres Erwerbspensum aufweisen (Epple et al. 2015). Gleichzeitig ist eine Erklärung anhand des Ansatzes der relativen Ressourcen ebenfalls denkbar, da Frauen mit höherer Bildung mehr Ressourcen zur Verfügung haben, die Hausarbeit an den Partner abzuschieben. Allerdings würde dies nur auf Personen zutreffen, die eine höhere Bildung als der Partner/die Partnerin haben. Hier wären weitere Analysen nötig, die das relative Verhältnis des Bildungsstandes beider Partner berücksichtigen.

Der Effekt der Bildung der PartnerInnen wirkt ebenfalls geschlechtsspezifisch. Frauen mit tertiär gebildeten Partnern leisten weniger Hausarbeit. Bei Männern zeigt sich hingegen, anders als erwartet, kein Zusammenhang. Sowohl der Ansatz der Zeitverfügbarkeit als auch der Ansatz der relativen Ressourcen kann diesen Zusammenhang nicht erklären, da sich zur Bestätigung beider Ansätze auch für Männer ein positiver Effekt zeigen müsste. Vielmehr kann der Zusammenhang als ein Indikator für den "doing gender" Ansatz betrachtet werden. Gleichzeitig haben Frauen mit höherer Bildung in der Mehrheit einen Partner, der ebenfalls über eine hohe Bildung verfügt (Blossfeld und Timm 1997). In diesen Haushalten ist eine egalitäre Arbeitsteilung sehr viel häufiger.

Wie erwartet zeigt sich ein positiver Zusammenhang zwischen einer Vollzeitbeschäftigung des/der PartnerIn und dem Zeitaufwand für Hausarbeit von beiden Geschlechtern. Dieses Ergebnis bestätigt den Ansatz der relativen Ressourcen. Männer leisten darüber hinaus mehr Hausarbeit, ist ihre Partnerin in Teilzeit beschäftigt, als Männer, deren Partnerin erwerbslos ist. Bei Frauen zeigt sich kein signifikanter Zusammenhang.

Die Modelle 3-5 für Frauen und 8-10 integrieren jeweils zwei kantonale Variablen. Innerhalb der Politik hat der Versorgungsgrad von Kinderbetreuungsplätzen 
für Frauen und Männer keinen signifikanten Zusammenhang mit dem Zeitaufwand für Hausarbeit. Hier kann die Alternativhypothese bestätigt werden. Weiter bestätigt sich die Annahme, wonach mit steigendem Anteil an Frauen im Parlament der Zeitaufwand für Hausarbeit sinkt. Dies trifft allerdings nur für Frauen zu.

Im Einklang mit den Erwartungen nimmt mit steigendem Anteil Deutschsprachiger in einem Kanton der Zeitaufwand für Hausarbeit von Männern ab. Hingegen reduziert sich entgegen den Erwartungen auch jener von Frauen. Beide Geschlechter leisten also in deutschsprachigen Kantonen weniger Hausarbeit.

Der Effekt von Traditionalismus bestätigt nur in dem Modell für Frauen die Hypothese. So leisten Frauen tendenziell mehr Hausarbeit, je traditioneller ein Kanton ist.

Die Variablen der Wirtschaftsdimension schliesslich zeigen für beide Geschlechter Zusammenhänge auf. So bestätigt sich hier die Hypothese, wonach Frauen in modernen und wirtschaftlich prosperierenden Kantonen eher in der Lage sind, ihren Aufwand für Hausarbeit zu reduzieren. Hingegen kann die Annahme für Männer nicht bestätigt werden, da sich ihr Zeitaufwand ebenfalls verringert.

Zusammenfassend lässt sich hinsichtlich des Einflusses von kantonalen Merkmalen Folgendes festhalten: Der Zeitaufwand für Hausarbeit von Frauen verringert sich mit steigendem Frauenanteil im Parlament, einem hohen Anteil Deutschsprachiger, zunehmender Beschäftigtenquote und steigender Urbanisierung. In traditionelleren Kantonen erhöht er sich hingegen. Die Hausarbeit von Männern sinkt mit steigendem Anteil Deutschsprachiger, steigender Beschäftigtenquote und zunehmender Urbanisierung.

Insgesamt zeigen die Ergebnisse, dass insbesondere der Zeitaufwand für Hausarbeit von Frauen relativ gut durch die integrierten Variablen, sowohl auf individueller als auch auf kantonaler Ebene, erklärt werden kann. Bei Männern zeigt sich ein anderes Bild. Hier sind es nur relativ wenige Variablen, die einen signifikanten Effekt haben. Diese geschlechtsspezifischen Unterschiede bei der Analyse von Zeitbudgetdaten sind allerdings nicht unüblich (Hook 2006; van der Lipp et al. 2011; Epple et al. 2015).

\section{Diskussion und Fazit}

Der vorliegende Artikel analysiert zum ersten Mal den Zusammenhang zwischen individuellen Merkmalen sowie kantonal variierenden politischen, kulturellen und wirtschaftlichen Faktoren und dem individuellen Zeitaufwand für Hausarbeit von erwerbstätigen Frauen und Männern, die in einer Partnerschaft leben. Die Ergebnisse verweisen auf die Bedeutung kantonaler Rahmenbedingungen für individuelles Handeln. Durch die hohe Autonomie der Kantone variieren politische und wirtschaftliche Faktoren erheblich. Diese historisch gewachsenen Unterschiede stehen 
dabei auch in engem Zusammenhang mit kulturellen Unterschieden. Die Analyse des Zeitaufwands für Hausarbeit stellt darüber hinaus die Wichtigkeit der Integration von unbezahlter Arbeit bei der Untersuchung von Geschlechterungleichheiten dar. Eine Vielzahl an Studien beleuchtet in diesem Zusammenhang insbesondere die Sphäre der Erwerbsarbeit. Die vorliegenden Ergebnisse verweisen aber darauf, dass diese einseitige Betrachtung zu kurz greift und auch die häusliche Sphäre von Relevanz ist. So betont auch Nancy Fraser (1994) in ihrem Entwurf der "dual earner/ dual careegiver society" die Feminisierung des männlichen Lebenslaufs, anstatt einer androzentrischen Gleichstellung.

Die Ergebnisse der Studie zeigen auf, dass es innerhalb der Schweiz gleichstellungsfördernde und gleichstellungshemmende Kontexte gibt. Als gleichstellungsfördernd wird dabei eine Reduktion des Zeitaufwands für Hausarbeit von Frauen und eine Erhöhung desjenigen von Männern verstanden. Umgekehrt wird von einem gleichstellungshemmenden Kontext gesprochen.

Kantone mit einem höheren Anteil an Frauen im Parlament stellen einen Kontext dar, in dem Frauen weniger Hausarbeit leisten als in anderen Kantonen. So kann der Frauenanteil im Parlament durchaus als Indikator für eine Politik verstanden werden, die der Gleichstellung der Geschlechter positiver gegenübersteht als in anderen Kantonen. Inwieweit eine stärkere Präsenz von Frauen im Parlament das Resultat oder der Auslöser einer moderneren Geschlechterkultur ist, bleibt hierbei allerdings offen. Der Versorgungsgrad an Kinderbetreuungsplätzen im Frühbereich, als politische Massnahme zur Vereinbarkeit von Familie und Beruf, erweist sich lediglich innerhalb der Erwerbsarbeit als gleichstellungsfördernder Kontext (Epple et al. 2015). Der ausbleibende Zusammenhang mit dem Zeitaufwand für Hausarbeit, insbesondere bei Frauen, ist möglicherweise auf den grossen Anteil an teilzeitbeschäftigten Frauen, insbesondere von Müttern, zurückzuführen (Stadelmann-Steffen 2007). So bleibt einem Grossteil der Frauen weiterhin genügend Zeit für Hausarbeit zur Verfügung.

Auch die kantonalen Unterschiede der kulturellen Dimension spielen eine wichtige Rolle hinsichtlich des Zeitaufwands für Hausarbeit. Doch die Trennung in gleichstellungsfördernde und -hemmende Kontexte ist etwas komplexer.

Die Romandie hat sehr viel früher als die restliche Schweiz für das Frauenstimmrecht votiert, ist aber bis zu dessen Einführung im Jahr 1971 an der Mehrheit der Nein-Stimmen gescheitert (Bühler 2002). Auch der Frauenanteil im Parlament ist in den Westschweizer Kantonen höher als in anderen Kantonen. Dennoch lässt sich die Grenze zwischen gleichstellungsfördernder beziehungsweise -hemmender Politik nicht ausschliesslich entlang der Sprachgrenze ziehen, da auch in den urbanen Kantonen der Deutschschweiz der Frauenanteil im Parlament hoch ist.

Auch das Ergebnis hinsichtlich des Zusammenhangs der Sprachregion lässt Zweifel aufkommen an der Einteilung: moderne Westschweiz gegenüber traditioneller Deutschschweiz. So ist der Zeitaufwand für Hausarbeit von Frauen und Männern 
in deutschsprachigen Kantonen geringer als in französisch- und italienischsprachigen Kantonen. Bühler und Heye (2005) und Pfau-Effinger (2005) beschreiben die französischsprachige Schweiz als fortschrittlicher hinsichtlich der Gleichstellung der Geschlechter. Sie machen dies besonders am Familienmodell fest, das häufiger als in der übrigen Schweiz aus dem Doppelverdiener-Modell besteht. Tatsächlich sind insbesondere bei Müttern die Erwerbsquoten und Erwerbsarbeitsstunden in der lateinischen Schweiz überdurchschnittlich hoch (Epple et al. 2015). Demnach überraschend ist das Ergebnis, dass sich diese positiven Aspekte hinsichtlich der Geschlechtergleichstellung nicht auch in die Hausarbeit übertragen haben, da nicht nur Männer, sondern auch Frauen weniger Hausarbeit in deutschsprachigen Kantonen leisten. Vielmehr kann eine gleichstellungsfördernde Politik und Kultur also nur bedingt mit einer Egalisierung der Hausarbeiten gleichgesetzt werden. Es ist anzunehmen, dass erwerbstätige Frauen insbesondere in französischsprachigen Regionen von einer Doppelbelastung durch Erwerbsarbeit und Hausarbeit betroffen sind.

Die Ergebnisse des zweiten Kultur-Indikators Traditionalismus belegen die Persistenz der klassischen geschlechtsspezifischen Aufgabenteilung in einigen Kantonen. Je traditioneller ein Kanton, umso mehr Hausarbeit leisten Frauen. Das Ergebnis validiert gleichzeitig auch die Eignung des Indizes als Indikator für die Geschlechterkultur. Die Variablen Traditionalismus und Sprachregion korrelieren relativ gering $(r=0.3)$, so dass hier ebenfalls jeweils unterschiedliche kulturelle Dimensionen gemessen werden. Die geringe Korrelation ist ein weiterer Hinweis auf eine Neuinterpretation der Rechnung: Französischsprachig geht einher mit höherer Gleichstellung. Das französischsprachige Genf ist beispielsweise neben dem deutschsprachigen Kanton Basel-Stadt der Kanton mit dem niedrigsten Grad an Traditionalismus, wohingegen das französischsprachige Wallis im oberen Mittelfeld liegt. Die höchsten Werte hinsichtlich des Traditionalismus haben allerdings deutschsprachige Kantone.

Die Resultate zeigen schliesslich, dass insbesondere auch wirtschaftliche Faktoren den Zeitaufwand für Hausarbeit beeinflussen. In modernen und wirtschaftlich prosperierenden Kantonen leisten Frauen und Männer deutlich weniger Hausarbeit. Es ist anzunehmen, dass sie aufgrund ihrer verstärkten Arbeitsmarktbeteiligung über eine bessere individuelle Verhandlungsmacht verfügen und so ihren Anteil reduzieren können. Es könnte auch postuliert werden, dass die Geschlechterkultur in diesen Kantonen eher offen gegenüber einer gleichberechtigten Arbeitsteilung der Hausarbeit ist. So nimmt auch der Traditionalismus-Index mit zunehmender Urbanisierung ab.

Neben kantonalen Faktoren wurde der Einfluss individueller Charakteristika getestet. Auch hier zeigen sich deutliche geschlechtsspezifische Unterschiede: Frauen mit einem hohen Erwerbspensum, hoher Bildung und hoch gebildeten Partnern leisten tendenziell weniger Hausarbeit. Verheiratete Frauen und Frauen mit einem vollzeiterwerbstätigen Partner leisten hingegen eher mehr Hausarbeit. Bei Männern 
reduziert ein hohes Erwerbspensum den Zeitaufwand für Hausarbeit, ein Kind, eine teilzeit- oder vollzeiterwerbstätige Partnerin oder eine externe Haushaltshilfe erhöhen hingegen den Zeitaufwand für Hausarbeit. Insgesamt können die Hypothesen weitgehend bestätigt werden. Gleichzeitig zeigen sie aber auch widersprüchliche Ergebnisse. So hat keine der Bildungsvariablen einen Zusammenhang mit der Hausarbeit von Männern. Überraschend ist auch das Ergebnis, wonach sich der Zeitaufwand für Hausarbeit von Männern noch erhöht, ist in ihrem Haushalt eine externe Haushaltshilfe beschäftigt. Möglicherweise betrifft das insbesondere Männer, die eine vollzeiterwerbstätige Partnerin haben und daher zusätzlich zur Haushaltshilfe anfallende Arbeiten übernehmen. Hier wären weitere Analysen nötig, die auch den Umfang der extern geleisteten Stunden integrieren könnten.

Die Analyse weist insgesamt einige Defizite auf. Die Studie fokussiert aufgrund der verfügbaren Daten zum kantonalen Vergleich auf die Individual-, nicht aber auf die Haushaltsebene. Es wären Informationen über den Zeitaufwand für Hausarbeit beider Partner wichtig, um den relativen Anteil an der gesamten Hausarbeit zu untersuchen (vgl. Knudsen und Wærness 2008). So konnten auch offengebliebene Fragen hinsichtlich der Dynamiken innerhalb von Haushalten nicht geklärt werden. Die Datenlage in der Schweiz erlaubt es allerdings derzeit nicht, die Haushaltsebene nach Kantonen zu analysieren.

Eine weitere Schwäche der Daten ist es, dass die relative Einflusskraft der Kontextfaktoren nicht gemessen werden konnte. So konnten pro Modell nur zwei Kontextfaktoren integriert werden und dies lässt die Frage offen, ob beispielsweise kulturelle Faktoren unter Kontrolle der Wirtschaftsfaktoren weiter Bestand haben.

Hinsichtlich der Gleichstellung der Geschlechter in der Schweiz lassen die Ergebnisse dieses Beitrags schliesslich zwei Schlüsse zu. Erstens muss der Fortschritt in der Gleichstellung in eine öffentliche und eine private Dimension (Walby 2004) eingeteilt werden. Zunehmende Gleichstellung auf dem Arbeitsmarkt lässt sich nicht per se auf die Arbeitsteilung im Haushalt übertragen. Doch gerade eine ungleiche Aufteilung der Hausarbeit in Paarhaushalten kann zu grossen Geschlechterunterschieden führen, wobei meist Frauen von der Doppelbelastung betroffen sind. Die zunehmende Frauenerwerbsquote ist daher nur die eine Seite der Medaille. Der Artikel macht deutlich, dass nur die Beleuchtung aller relevanten Bereiche, in denen gearbeitet wird, auch ein umfassendes Bild von Geschlechtergleichheit liefert. Zweitens macht gerade der Vergleich der Kantone deutlich, dass die Dichotomisierung der Schweiz - gleichstellungsaffine Westschweiz und konservative Deutschschweiz nicht mehr aufrecht erhalten werden kann. Vielmehr wird die Komplexität der Zusammenhänge zwischen politischen und gesellschaftlichen Rahmenbedingungen und der geschlechtsspezifischen Arbeitsteilung deutlich. Die Gleichstellung der Geschlechter in der Schweiz stellt demnach eine diffizile Aufgabe dar, der sich eine Vielzahl an gesellschaftlichen Akteuren stellen muss. 


\section{Literaturverzeichnis}

Armingeon, Klaus, Fabio Bertozzi und Giuliano Bonoli. 2004. Swiss worlds of welfare. West European Politics 27(1): 20-44.

Batalova, Jeanne A. und Philip N. Cohen. 2002. Premarital cohabitation and housework: Couples in cross-national perspective. Journal of Marriage and Family 64(3): 743-755.

Becker, Gary S. 1991. A treatise on the family. Cambridge, MA: Harvard University Press.

BFS (Bundesamt für Statistik). 2009. Schweizerische Arbeitskräfteerhebung (SAKE). Erhebung des Bundesamts für Statistik. Neuenburg: BFS.

BFS (Bundesamt für Statistik). 2010. Schweizerische Arbeitskräfteerhebung (SAKE). Erhebung des Bundesamts für Statistik. Neuenburg: BFS.

Bianchi, Suzanne M. und Melissa A. Milkie. 2010. Work and family research in the first decade of the 21st century. Journal of Marriage and Family 72(3): 705-725.

Bianchi, Suzanne M., Melissa A. Milkie, Linda C. Sayer und John P. Robinson. 2000. Is anyone doing the housework? Trends in the gender division of household labor. Social Forces 79(1): 191-228.

Blossfeld, Hans-Peter und Andreas Timm. 1997. Der Einfluß des Bildungssystems auf den Heiratsmarkt. Eine Längsschnittanalyse der Wahl des ersten Ehepartners im Lebenslauf. Kölner Zeitschrift für Soziologie und Sozialpsychologie 49(3): 440-476.

Bonoli, Guliano und Benoît Gay-des Cômbes. 2005. Travail à temps partiel masculin et engagement des hommes dans les tâches domestiques et familiales. Schweizerische Zeitschrift für Soziologie 31(1): 145-166.

Buchmann, Marlis. 2002. Halb drinnen - halb draussen. Analysen zur Arbeitsmarktintegration von Frauen in der Schweiz. Chur: Rüegger.

Buchmann, Marlis, Irene Kriesi und Stefan Sacchi. 2010. Labour market structures and women's employment levels. Work, Employment \& Society 24(2): 279-299.

Bühler, Elisabeth. 2002. Formen der Vereinbarkeit von Erwerbsarbeit und Familie. Strukturen und Entwicklungstendenzen in der Schweiz. Geographische Zeitschrift 90(3/4): 167-179.

Bühler, Elisabeth und Corinna Heye. 2005. Fortschritte und Stagnation in der Gleichstellung der Geschlechter 1970-2000. Neuenburg: Bundesamt für Statistik.

Bühler, Elisabeth und Verena Meier Kruker. 2002. Gendered labour arrangements in Switzerland: Structures, cultures, meanings: statistical evidence and biographical narratives. GeoJournal 56(4): 305-313.

Bühlmann, Jacqueline und Beat Schmid. 1999. Unbezahlt - aber trotzdem Arbeit. Zeitaufwand für Haus- und Familienarbeit, Ehrenamt, Freiwilligenarbeit und Nachbarschaftshilfe. Neuenburg: BFS.

Coltrane, Scott. 2000. Research on household labor: Modeling and measuring the social embeddedness of routine family work. Journal of Marriage and Family 62(4): 1208-1233.

Cooke, Lynn Prince. 2007. Policy pathways to gender power: State-level effects on the US division of housework. Journal of Social Policy 36(2): 239-260.

Cooke, Lynn Prince. 2011. Gender-class Equality in Political Economies. London: Routledge.

Coverman, Shelley. 1985. Explaining husbands' participation in domestic labor. Sociological Quarterly 26(1): 81-97.

Cyba, Eva. 2000. Geschlecht und soziale Ungleichheit. Konstellation der Frauenbenachteiligung. Opladen: Leske und Budrich.

Davis, Shannon N., Theodore N. Greenstein und Jennifer P. Gerteisen Marks. 2007. Effects of union type on division of household labor: Do cohabiting men really perform more housework? Journal of Family Issues 28(9): 1246-1272. 
Epple, Ruedi. 2013. Die Berechnung des Traditionalismus-Index. Working Paper, http://lettres.unifr.ch/ fileadmin/Documentation/Departements/Sciences_sociales/Soziologie_Sozialpolitik_und_Sozialarbeit/Forschung/NFP60/Trad_Index.pdf (15.10.2013).

Epple, Ruedi, Martin Gasser, Sarah Kersten, Michael Nollert und Sebastian Schief. 2014. Institutions and gender time inequality: A fuzzy-set QCA of Swiss cantons. Schweizerische Zeitschrift für Soziologie 40(2): 259-278.

Epple, Ruedi, Martin Gasser, Sarah Kersten, Michael Nollert und Sebastian Schief. 2015. Parenthood and employment: The impact of policies and culture on gender inequality in Switzerland. Equality, Diversity and Inclusion: An International Journal 34(2): 141-154.

Esping-Andersen, Gøsta. 1990. The Three Worlds of Welfare Capitalism. Princeton, NJ: Princeton University Press.

Fraser, Nancy. 1994. After the family wage: Gender equity and the welfare state. Political Theory 22(4): 591-618.

Fuwa, Makiko. 2004. Macro-level gender inequality and the division of household labor in 22 countries. American Sociological Review 69(6): 751-767.

Gasser, Martin und Sarah Kersten. 2015. Zeit für Kinder? Väterliches Engagement in der Schweiz. In Unbezahlte Arbeit, hrsg. von Monica Budowksi und Michael Nollert. Zürich: Seismo.

Gasser, Martin, Sarah Kersten, Michael Nollert und Sebastian Schief. 2015. Geschlechtsspezifische Ungleichheiten in der bezahlten und unbezahlten Arbeit: Kantonale Muster der Zeitungleichheit. Schweizerische Zeitschrift für Soziologie 41(1): 9-31.

Geist, Claudia. 2005. The welfare state and the home: Regime differences in the domestic division of labour. European Sociological Review 21(1): 23-41.

Gershuny, Jonathan und Oriel Sullivan. 2003. Time use, gender, and public policy regimes. Social Politics: International Studies in Gender, State \& Society 10(2): 205-228.

Gornick, Janet C. und Marcia K. Meyers. 2004. Welfare regimes in relation to paid work and care. S. 45-67 in Changing Life Patterns in Western Industrial Societies, hrsg. von Janet Zollinger Giele und Elke Holst. Amsterdam: Elsevier.

Gupta, Sanjiv. 2007. Autonomy, dependence, or display? The relationship between married women's earnings and housework. Journal of Marriage and Family 69(2): 399-417.

Gupta, Sanjiv, Marie Evertsson, Daniela Grunow, Magnus Nermo und Liana C. Sayer. 2010. Economic inequality and housework. S. 105-124 in Dividing the Domestic. Men, Women, and Household Work in Cross-national Perspective, hrsg. von Judith Treas und Sonja Drobnič. Stanford, CA: Stanford University Press.

Hans Böckler Stiftung. 2014. Doppelt belastet bis zur Erschöpfung. Böcker Impuls 04, http://www. boeckler.de/impuls_2014_04_gesamt.pdf (07.03.2014).

Hofstede, Geert H. 2001. Culture's Consequences. Comparing Values, Behaviors, Institutions, and Organizations Across Nations. Second edition. Thousand Oaks, CA: Sage Publications.

Hook, Jennifer L. 2006. Care in context: Men's unpaid work in 20 countries, 1965-2003. American Sociological Review 71(4): 639-660.

Hox, Joop J. 2010. Multilevel Analysis. Second edition. London: Routledge Academic.

IDHEAP/BADAC. 2014. BADAC - Zustand des Staates auf Niveau Schweizerische Kantone. http:// www.badac.ch/db/db.php?intro=cantons\&lang=De (10.02.2014).

Inglehart, Ronald und Pippa Norris. 2003. Rising Tide. Gender Equality and Cultural Change Around the World. Cambridge, UK und New York: Cambridge University Press.

Knudsen, Knud und Kari Wærness. 2008. National context and spouses' housework in 34 countries. European Sociological Review 24(1): 97-113.

Korpi, Walter. 1983. The Democratic Class Struggle. London und Boston: Routledge \& K. Paul. 
Künzler, Jan, Wolfgang Walter, Elisabeth Reichart und Gerd Pfister. 2001. Gender division of labour in unified Germany. European Network on Policies and the Division of Unpaid and Paid Work (WORC Report), http://www.politikwissenschaft.uni-wuerzburg.de/fileadmin/06060101/na_rep. $\operatorname{pdf}(12.01 .2012)$.

Pfau-Effinger, Birgit. 2000. Kultur und Franenerwerbstätigkeit in Europa: Theorie und Empirie des internationalen Vergleichs. Opladen: Leske + Budrich.

Pfau-Effinger, Birgit. 2005. Welfare state policies and the development of care arrangements. European Societies 7(2): 321-347.

Phillips, Anne. 1995. The Politics of Presence. Oxford: Clarendon.

Rabe-Hesketh, Sophia und Anders Skrondal. 2005. Multilevel and Longitudinal Modeling Using Stata. College Station, TX: Stata Press.

Sayer, Liana C. 2010. Trends in housework. S. 19-40 in Dividing the Domestic. Men, Women, and Household Work in Cross-national Perspective, hrsg. von Judith Treas und Drobnič. Stanford, CA: Stanford University Press.

Schmidt, Manfred G. 1997. Vergleichende Policy-Forschung. S. 261-276 in Vergleichende Politikwissenschaft, hrsg. von Dirk Berg-Schlosser und Ferdinand Müller-Rommel. Wiesbaden: VS Verlag für Sozialwissenschaften.

Schön-Bühlmann, Jaqueline und Carole Liechti. 2013. Das Engagement der Väter in Haushalt und Familie. Neuenburg: BFS.

Snijders, Tom und Roel J. Bosker. 2011. Multilevel Analysis. An Introduction to Basic and Advanced Multilevel Modeling. Los Angeles: Sage.

Stadelmann-Steffen, Isabelle. 2007. Policies, Frauen und der Arbeitsmarkt. Die Frauenerwerbstätigkeit in der Schweiz im internationalen und interkantonalen Vergleich. Zürich und Münster: LIT Verlag.

Stern, Susanne, Rolf Iten, Stephanie Schwab, Christina Felfe, Michael Lechner und Petra Thiemann. 2013. Familienergänzende Kinderbetreuung und Gleichstellung. Zürich/St. Gallen: INFRAS/ Schweizerisches Institut für Europäische Wirtschaftsforschung.

Stier, Haya und Noah Lewin-Epstein. 2007. Policy effects on the division of housework. Journal of Comparative Policy Analysis: Research and Practice 9(3): 235-259.

Stolz, Jörg. 2001. Traditionalismus und das Fremde: Einstellungen zu Ausländern und Ausländerinnen 1995. S. 81-105 in Das Fremde in der Schweiz. Ergebnisse soziologischer Forschung, hrsg. von Hans-Joachim Hoffmann-Nowotny. Zürich: Seismo.

Sullivan, Oriel. 2013. What do we learn about gender by analyzing housework separately from child care? Some considerations from time-use evidence. Journal of Family Theory \& Review 5(2): 72-84.

Treas, Judith und Sonja Drobnič. 2010. Dividing the Domestic. Men, Women, and Household Work in Cross-national Perspective. Stanford, CA: Stanford University Press.

UNDP (United Nations Development Program). 2009. Human Development Report. New York: UNDP.

van der Lippe, Tanja, Judith de Ruijter, Esther de Ruijter und Werner Raub. 2011. Persistent inequalities in time use between men and women: A detailed look at the influence of economic circumstances, policies, and culture. European Sociological Review 27(2): 164-179.

Vatter, Adrian. 2002. Kantonale Demokratien im Vergleich. Entstehungsgründe, Interaktionen und Wirkungen politischer Institutionen in den Schweizer Kantonen. Opladen: Leske + Budrich.

Walby, Sylvia. 2004. The European Union and gender equality: Emergent varieties of gender regime. Social Politics: International Studies in Gender, State \& Society 11(1): 4-29.

West, Candice und Zimmerman Don H. 1987. Doing gender. Gender and Society 1(2): 125-151. 


\section{Michael Nollert und Amir Sheikhzadegan} (Hrsg.)

\section{Gesellschaften \\ zwischen \\ Multi- und \\ Transkulturalität}

208 Seiten

ISBN 978-3-03777-151-8

SFr. 38.-/Euro 34.-

Die zunehmende Immigration aus islamisch geprägten Staaten stellt eine kulturelle und sozialpolitische Herausforderung dar. Kulturelle Differenz wird dabei unter unterschiedlichen theoretischen Prämissen diskutiert: Vertreterlnnen des Multikulturalismus gehen davon aus, dass ein Zusammenleben verschiedener Kulturen nicht nur wünschbar, sondern auch möglich ist. "Transkulturalität» impliziert hingegen, dass Kulturen keine homogene, abgrenzbare Entitäten sind, und es im Hinblick auf soziale Kohäsion auch nicht wünschbar ist, dass soziale Gruppen kulturelle Differenzen akzentuieren oder gar politisch instrumentalisieren.

Michael Nollert ist Professor für Sozialforschung und Sozialpolitik im Studienbereich Soziologie, Sozialpolitik und Sozialarbeit der Universität Fribourg.

Amir Sheikhzadegan ist Doktorassistent im Studienbereich Soziologie, Sozialpolitik und Sozialarbeit der Universität Fribourg. 


\title{
Des hommes en mouvement en Suisse: trois perspectives sur la masculinité
}

\author{
Hakim Ben Salah*, Jean-Martin Deslauriers * ${ }^{*}$ et René Knüsel*
}

\section{Introduction}

Cet article présente et analyse trois perspectives sur la masculinité. Cette expression désigne un ensemble de points de vue sur la situation sociale et politique des hommes au sein d'une société (Clatterbaugh 1997). Les perspectives en question ont émergé d'une analyse de contenu des discours diffusés par des hommes en mouvement, c'est-à-dire un ensemble d'organisations réunissant des hommes consciemment impliqués dans des activités visant à (re)définir la place et le rôle de l'homme (Ben Salah 2013). L'étude présentée dans cet article - d'envergure nationale et réalisée entre 2009 et 2013 - expose les résultats issus de deux phases de récoltes de données. Les développements présentent en priorité les résultats d'une analyse de contenu réalisée sur les sites de 40 organisations jugées représentatives des principales écoles de pensée identifiées, et intègre, dans une moindre mesure, les résultats d'analyses issues d'une enquête par questionnaire diffusée auprès des membres des 103 organisations répertoriées.

Cet article présente en introduction quelques éléments significatifs permettant de relier les perspectives sur la masculinité aux changements intervenus ces dernières décennies en Suisse, en regard de la configuration des rapports sociaux entre hommes et femmes. La section méthodologique expose ensuite la façon dont les organisations masculines ont été sélectionnées et la manière dont les données ont été récoltées et analysées. La partie des résultats présente, successivement, chacune des trois perspectives sur la masculinité. Elles font ensuite l'objet d'une discussion, à la fois en référence aux éléments de contexte présentés en introduction, ainsi que sur le plan de leur potentiel d'action et de mobilisation. Enfin, la conclusion revient sur les principaux acquis de cet article.

Les regards sur la condition féminine et masculine peuvent porter aussi bien sur les plans politique, économique que culturel ou légal (Bunge 2003). Sous ses différents aspects, bien qu'encore largement à l'avantage des hommes, la situation suisse présente un bilan contrasté.

L'égalité économique entre les sexes n'est pas encore atteinte, puisqu'à qualification égale les femmes sont toujours moins bien payées que les hommes,

\footnotetext{
* Université de Lausanne, CH-1015 Lausanne, hakim.bensalah@unil.ch et rene.knusel@unil.ch.

** Université d'Ottawa, CA- K1N 6N5 Ottawa, jean-martin.deslauriers@uottawa.ca.
} 